\title{
Conceptual design of IR multi-IFU spectrograph with MOAO
}

\author{
Daigo Tomono $^{a}$, Wolfgang Gæssler ${ }^{b}$, and Tetsuo Nishimura ${ }^{a}$ \\ ${ }^{a}$ Subaru Telescope, National Astronomical Observatory of Japan, 650 North Aohoku Place, \\ Hilo, Hawaii 96720, U.S.A.; \\ ${ }^{b}$ Max-Planck-Institute for Astronomy, Kœnigstuhl 17, D-69117 Heidelberg, Germany
}

\begin{abstract}
To study properties of cold dark matter (CDM), which can only be observed through its gravitational interaction with galaxies, spatially resolved spectra at least to the K-band are desirable. We started designing a spectrograph which observes multiple targets spatially resolved in a telescope field of view fed with multi-object adaptive optics (MOAO). The current design either places field lenses on the telescope field of view to image the pupil onto steering mirrors, or uses a single set of field lens to deliver beams to pick-off arms. The steering mirror on the pupil image tilts and selects a sub-field from each of the telescope field of view physically split by the field lenses. This allows cheaper and more robust construction of a method to select the target fields with a limitation in selections of the target fields. On the other hand, the pick-off arm implementation allows more flexibility in assigning targets to fields of the integral field units (IFUs) especially when targets are clustered. The IFU arranges spatial elements of each of sub-field of view to be fed into the spectrograph. If enough pixels are afforded, using microlens arrays, which image pupils of spatial elements onto the object plane of the spectrograph is ideal in robustness. Otherwise, an image slicer is to be located to arrange the sub-field of view onto the entrance slit. The instrument should be built as modules to allow expeditious scientific results.
\end{abstract}

Keywords: Subaru Telescope, Adaptive Optics, Infrared spectroscopy, Multi-object spectroscopy, Integral field unit, 3-dimensional spectroscopy

\section{INTRODUCTION}

As the study of the universe rapidly fills the space-time between present and the very beginning through observations using wide-field cameras and multi-object spectrographs, it is becoming more plausible that cold dark matter (CDM) controls many aspects of the material universe - origin, evolution, interaction, and current structure of galaxies, clusters and superclusters. Thus, study of CDM would be one of the major topics in the next decade. The elusive CDM seems to manifest only through the gravitational interaction with galaxies such as rotation curves of galaxies, kinematics of galaxies within clusters, galaxy mergers, and in some cases in gravitational lensing. We propose Conceptual Infrared Multi-Object Spectrograph (CIRMOS) precisely for the purpose. The capability of CIRMOS, however, with its versatility of multiple integral field units (IFUs) would not be limited to the study of CDM controlled evolution we are proposing as our science case. The final configuration of CIRMOS, as it is being conceived at this time, would be multiple IFU deployable to the entire field of six arc-minutes diameter at the Cassegrain focus of Subaru Telescope with $J$ to $K$-band spatial resolution enhancement by multi-object adaptive optics (MOAO) controlled with multiple laser guide stars (LGSs).

\section{BASELINE PARAMETERS}

In addition to basic design parameters of an infrared instrument, like spatial and spectral resolutions, a multiIFU instrument has a number of extra parameters to be defined. Figure 1 shows dependencies between the parameters of such an instrument and our view of how such instrument project may be achieved.

Further author information: (Send correspondence to D.T..)

D.T.: E-mail: tomono at subaru.naoj.org, Telephone +1 8089345974

Copyright 2008 Society of Photo-Optical Instrumentation Engineers.

This paper was (will be) published in Ground-based and Airborne Instrumentation for Astronomy II, Proceedings of the SPIE, Volume 7014 and is made available as an electronic reprint (preprint) with permission of SPIE. One print or electronic copy may be made for personal use only. Systematic or multiple reproduction, distribution to multiple locations via electronic or other means, duplication of any material in this paper for a fee or for commercial purposes, or modification of the content of the paper are prohibited. 


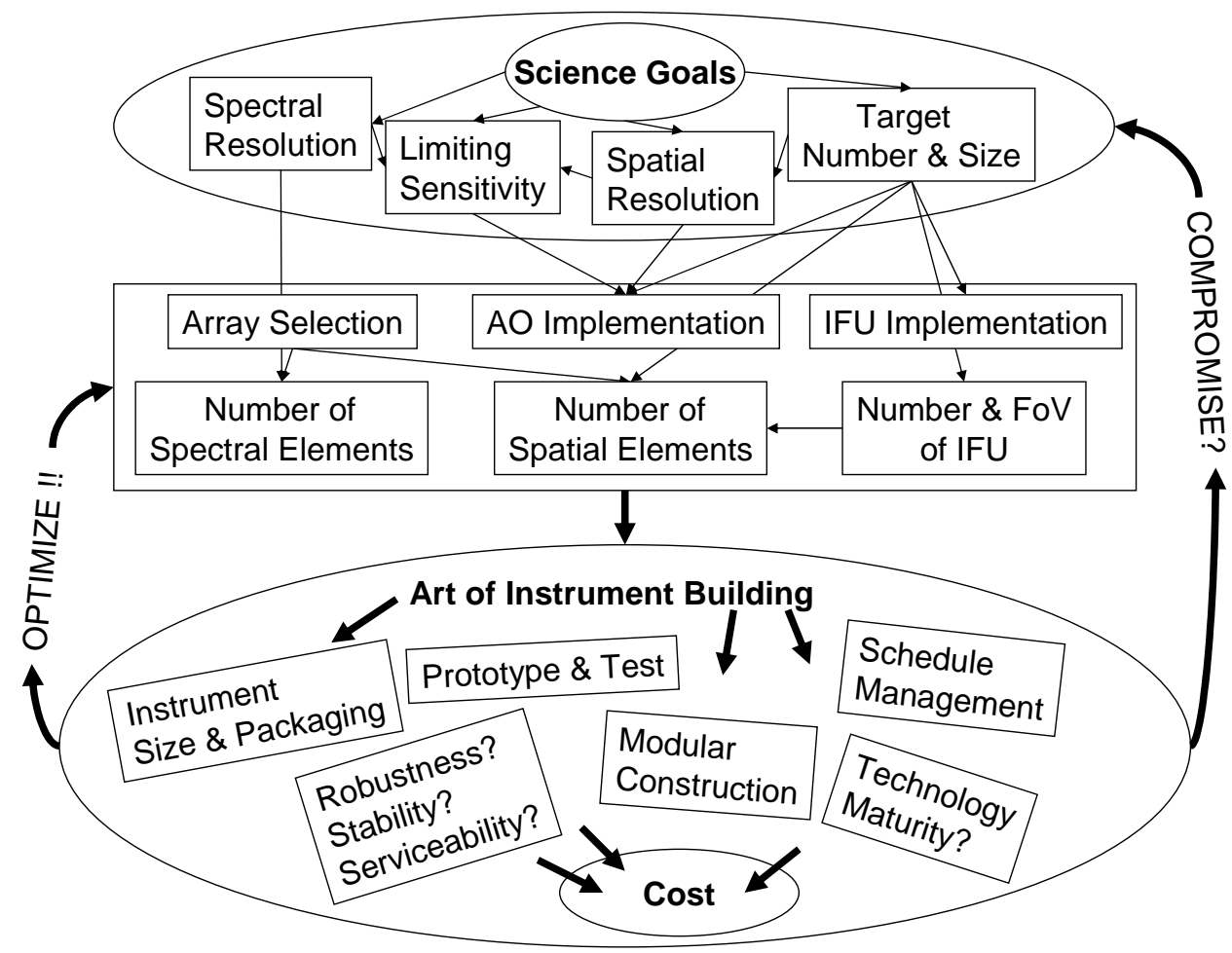

Figure 1. Relations between main parameters of a multi-IFU spectrograph. The direction of the arrows show dependency of parameters.

Among the parameters, number of targets is dependent upon sensitivity. Figure 2 shows numbers of interesting sources in a $\phi 6^{\prime}$ field. An estimate ${ }^{1}$ shows that the $5 \sigma$ sensitivity with 6 hours on-source integration is $20-21$ magnitudes in the $K$-band, which delivers around, e.g., 10 high-z targets.

Spatial resolution and spectral resolution should be optimized for scientific goals. Figure 3 shows existing and planned instruments on a parameter space defined with spectral resolution, spatial resolution, and field of view of the IFU for each target. We assumed the typical sizes of the galaxies to be $\sim 0.5^{\prime \prime}$ for $z>1$ and $\sim 0.25^{\prime \prime}$ for $z>2$. To detect those galaxies, spatial resolution of less than the typical size is optimal to reduce noises. On the other hand, to determine dynamics inside the galaxies, spatial resolution should be high enough to resolve velocity changes within a galaxy. The same discussion also applies to spectral resolution. In the figure, we defined that $\mathrm{R} \sim 3000$ is needed to determine dynamics inside the galaxies. Our goal is to study CDM through dynamics. It requires spectral resolution high enough to determine dynamics while maintaining good sensitivity. Spatial resolution of the instrument should be as high as possible, with less requirement for high dynamic range capabilities. While the details being described in the following sections, we have defined the parameters shown in Table 1 as the baseline.

\section{PROJECT STRATEGY}

Although the goal is ambitious, the approach we consider is that of "divide and conquer" by proposing to structure the project with modularized prototyping concepts to solve engineering challenges by steps. By this approach we intend to shift major budgetary burden toward the very end of the project, avoiding complex processes inherent to high cost projects, and at the same time reducing the technical risks.

The final configuration of CIRMOS utilizes MOAO on each IFU field. Currently a concept is under study to develop a single scientific module concurrently with development of tomographic technology for eventual MOAO 


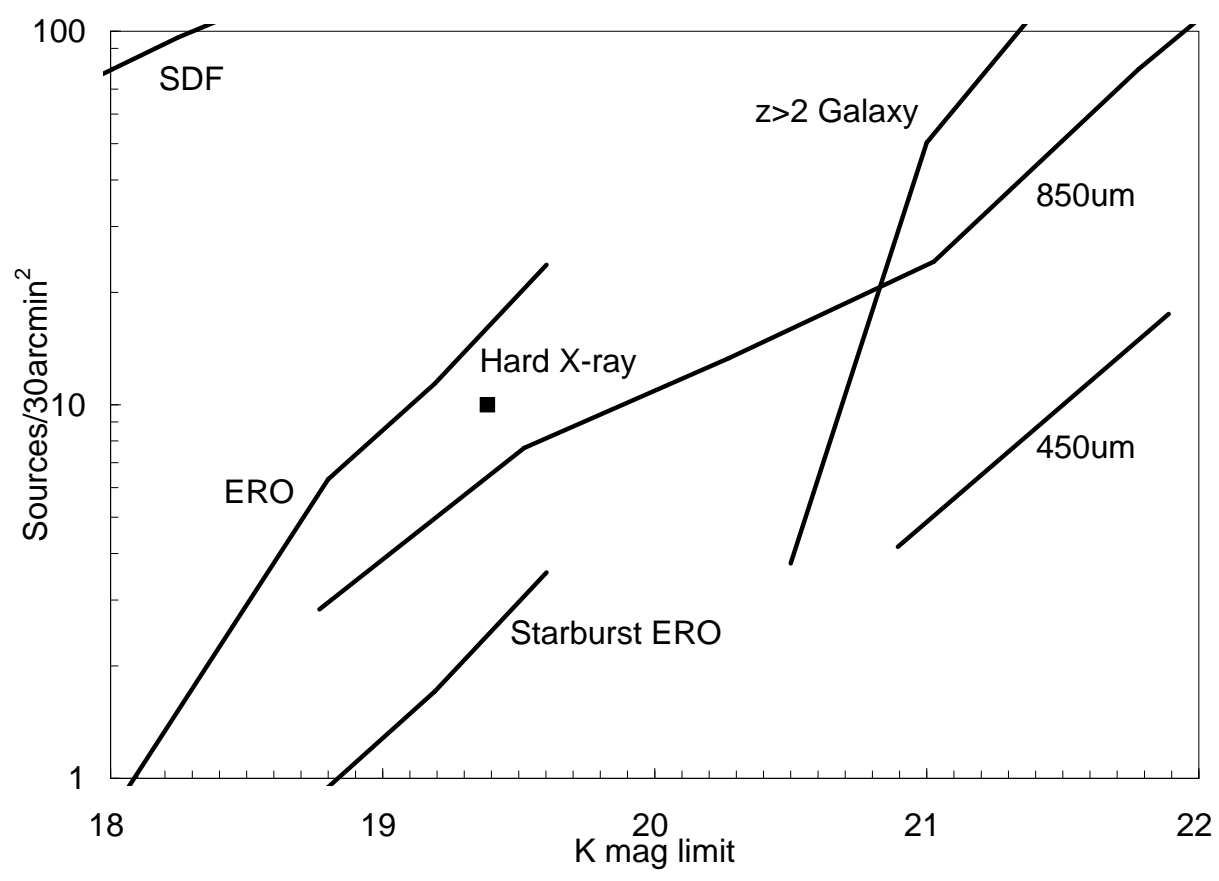

Figure 2. Number densities of extragalactic sources from the literature: 450 and $850 \mu$ m sources from Ref. 2, EROs and Starburst EROs from Ref. 3, z>2 galaxies from Ref. 4, SDF galaxies from Ref. 5, and Hard X-ray sources from Ref. 6.

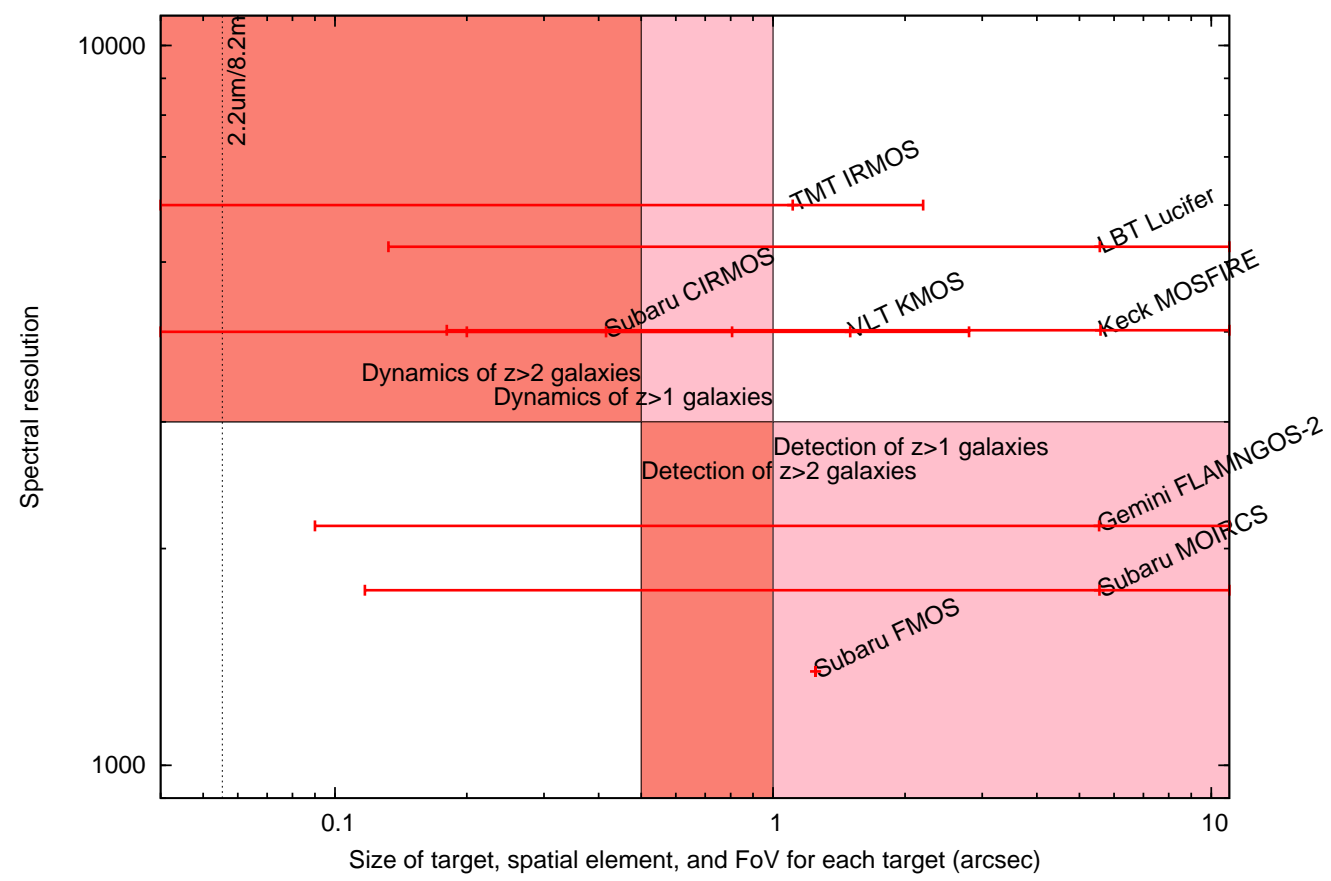

Figure 3. Parameters of other multi object IR spectrographs shown with scientific goals on the parameter space. Horizontal bar for each instrument shows the spatial resolution at the left and FoV for each target at the right. 
Table 1. Baseline parameters of the instrument

\begin{tabular}{cc}
\hline Accessible field of view & $\phi 6^{\prime}$ \\
\hline Wavelengths coverage & $J, H$, and $K$-bands, optimized at $K$-band \\
AO & MOAO with optional GLAO \\
IFU & Image slicer or microlens array \\
Spectral resolution & 4000 \\
Spatial resolution & $0.05^{\prime \prime}$ \\
Field of view of each IFU & $1^{\prime \prime}$ \\
Number of IFUs & 8 or 16 \\
\hline
\end{tabular}

(Table 2). The MOAO component may be incorporated in a module later in the form of cryogenic micro-electromechanical systems (MEMS).

In the following, the conceptual design is discussed from three aspects: science modules which are optmechanics for acquiring scientific spectra, adaptive optics (AO), and modular construction of these optmechanics.

\subsection{Options on Science Modules}

In designing a near infrared (NIR) multi-IFU instrument, there are a number of options to be considered even just for the science optical path. Assuming to be mounted on Subaru Telescope, Cassegrain focus is the best. Selection of the targets on the telescope field of view (FoV) can be done either using pick-off arms, with more flexibility, or steering mirrors in split fields, with more durability. Selection shall be made according to scientific requirements. After selecting targets, the image has to be arranged so that the spectrograph can acquire spectra of each spatial element. Microlens arrays (MLAs) allow simpler optics while requiring more number of pixels on focal plane array (FPA) than image slicers.

\subsubsection{Selection of Focus}

On Subaru Telescope, we have a choice of prime, Nasmyth, or Cassegrain focus where the instrument is to be mounted. Among these, Cassegrain focus is the best for CIRMOS. For instruments like CIRMOS, the prime focus is not adequate because of its short focal length which makes plate scale too small for physical limitations of the IFU. On the Nasmyth focus, the telescope has a field of view of $\phi 3.5^{\prime}$ with the image derotator or $\phi 6.0^{\prime}$ without it, both limited by mechanisms around the focus. The required accessible field of view can be achieved when the image derotator is not used. However, field rotation during exposures has to be taken care of with one of the following methods. (1) A dedicated image derotator with enough field of view. This requires a re-imaging optics between the telescope and the derotator. The re-imaging optics and the derotator can be huge to cover the field of view. (2) An instrument rotator. With the limited ceiling height over the Nasmyth platform of the telescope, the instrument has to be rotated around a horizontal axis. The instrument has to be as rigid as it were to be mounted at the Cassegrain focus.

On the Cassegrain focus, the telescope has a field of view of $\phi 6.8^{\prime}$ without the atmospheric dispersion corrector, limited by mechanisms around the focus. This allows the instrument to access the required $\phi 6.0^{\prime}$ field. Because the field rotation during exposures is compensated with the instrument rotator, the instrument has to be rigid enough.

\subsubsection{Pick-off Arms or Split Fields}

To select targets over the telescope field of view, there are two methods currently considered. First is to insert pick-off arms, each of which has a set of optics to retrieve image from desired sub-field of the desired target and re-image it onto the corresponding IFU. Second is to physically divide the telescope field of view into sub-fields. On each field, the entrance pupil is imaged onto a mirror that is tilted to select the target within corresponding sub-field of view. The former has more flexibility in selecting targets to be observed, especially when the targets are clustered. On the other hand, the latter requires less number of moving mechanisms, making the instrument more durable and cheaper. 


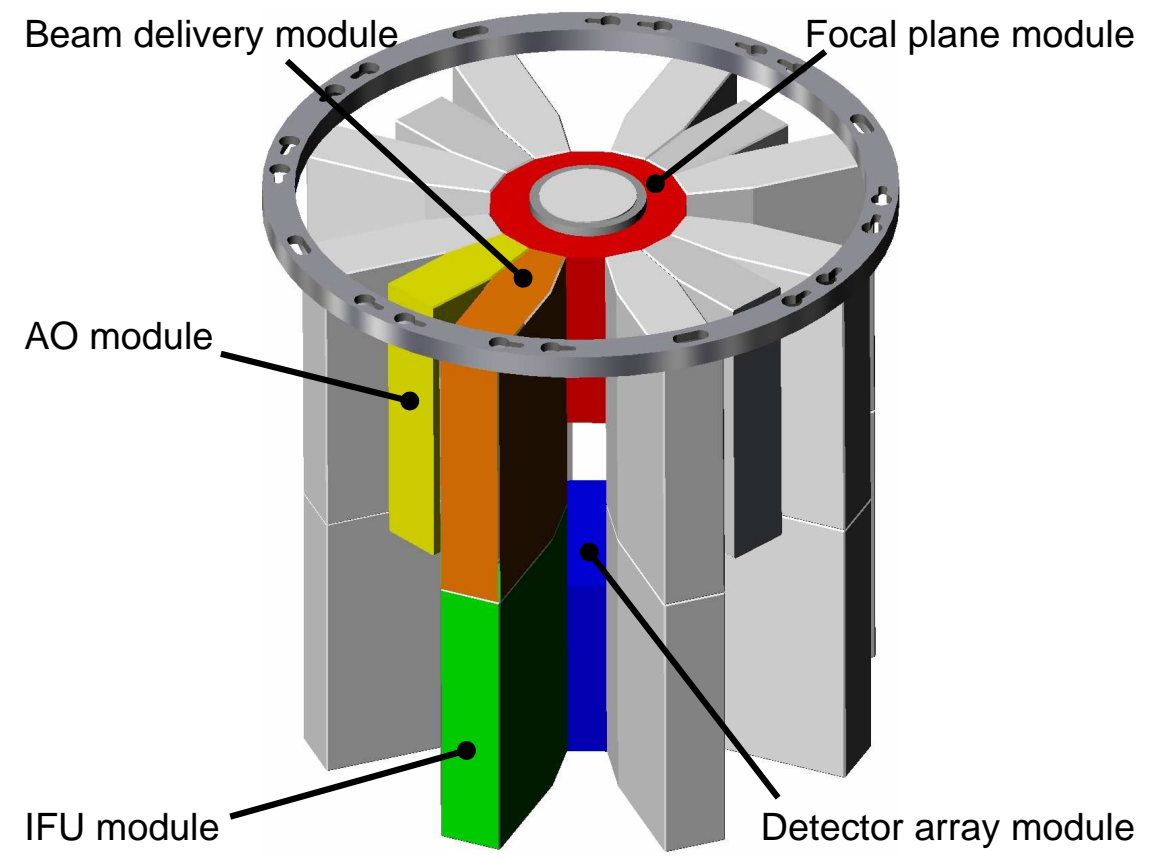

Figure 4. Scientific modules as well as AO modules on the Cassegrain focus. The light from the telescope is delivered from the top of the drawing. Focal plane module has either a window to cover the whole FoV in the pick-off arms option or physically divided windows in the split fields option. The light is picked up and delivered to the beam delivery modules. After sub-fields are selected, IFUs in the IFU modules arrange the field so the spectrograph(s) and FPA(s) inside the detector array module acquire spatially resolved spectra.

Pick-off Arms Target science may require sample fields that feed to each IFU placed as close as possible to each other. Configurations with pick-off mirrors on manipulator arms on or close to the telescope focal plane have been already considered by several groups. It is sometimes called fishermen-round-the-pond arrangement and is discussed briefly here. Versions of this configuration are investigated by KMOS, and also the TMT projects.

A small pick-off mirror is located at the tip of the arm and pivoted at a point on a perimeter that surrounds the focal plane so that the mirror reaches the entire field by the combination of rotational and radial movements. In one of our possible configurations, the pivoted arm carries a second collimation mirror and a third folding mirror on the pivot axis that brings the collimated beam vertically out from the pivoted arm and along the pivot axis. The beam that exits from the pivot arm is a collimated beam and can be directed to any direction by fixed optical components also on the pivot axis. Before the collimated beam refocus onto the IFU entrance plane, we may insert a field-rotation optics, and possibly up to two deformable mirrors (DM) for AO in the collimated section.

The module that contains a pivoted arm, collimated and refocusing sections can be called beam delivery (BD) module and may sit on top of an IFU module, as shown in Figure 4. The pick-off mirrors do not have to be on the focal plane and can be staggered vertically to increase the proximity of two or more sample fields. The sample fields may not join to each other, but could be very close this way. A possible BD module may contain two such pivoted arms that operate independently.

The conceptual arrangement using this scheme may have eight such modules circularly arranged around the telescope focal plane with total 16 pick-off mirrors. How closely these 16 pick-off mirrors can be brought together to each other for close-pack sample field situation has to wait detailed design. Further, for operation, optimum field configuration would require high level of optimization program to control arm arrangement with consideration for mechanical interference between arms.

This pick-off mirror scheme can be configured to sample a natural or laser guide star to a similar module with a wave-front sensor. 


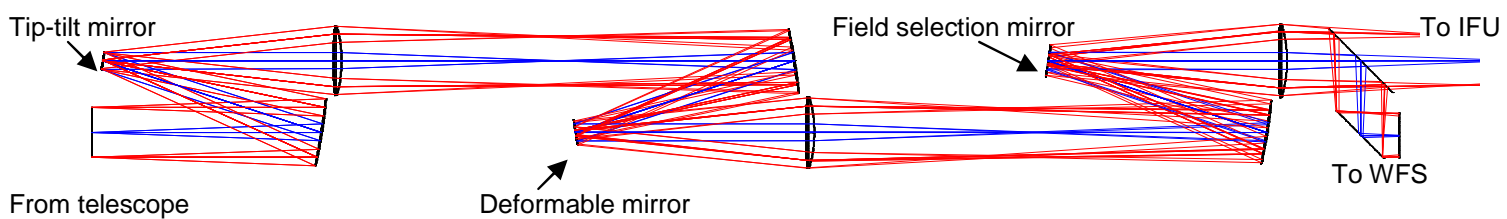

Figure 5. Optical train of the split field option. A set of optics is shown to select a target to be observed and re-image it onto the corresponding IFU. Light from the target as well as the reference for AO is supplied from the left over a split field of about $1^{\prime}$. The mirror images the entrance pupil onto the tip-tilt mirror and the lens re-images the field. Another re-imaging optics consists of a DM to correct for wavefront errors. The third re-imaging optics has a field selection mirror on the re-imaged pupil image. The mirror selects the target inside the split field to be fed to the IFU. A dichroic mirror, which also can be steered, reflects the visible light onto the wavefront sensor to acquire a reference light source in the same $1^{\prime}$ split field.

Split fields This option uses physically separated optics for each IFU which physically splits the telescope field of view into smaller sub fields of an order of $1^{\prime}$. We expect this alternative to be more reliable and cheaper than the pick-off arm option, with much less flexibility in assigning IFUs to targets to be observed. This would be an alternative, if the scientific requirements does not justify the cost to overcome the technical challenges associated with the pick-off arms.

Figure 5 shows a sample design of a set of optics from a split field to to the corresponding IFU including components for AO. The optics selects the target with steering a mirror on the pupil image plane. By placing the field selection mirror on the pupil image plane, steering of the mirror only affects the position of the re-imaged target without changing orientation of the rays. In the figure, a tip-tilt mirror and a DM for AO are added.

Filling factor of the sub-fields where targets can be acquired is about half. As seen in Figure 5, re-imaging optics occupies a part of telescope field of view making field areas where targets can not be observed. Optimization in opt-mechanical design is needed to maximize the filling factor.

Requirement to the steering mirror mechanism is not strict. For an example, to select a target with accuracy corresponding to half a spatial resolution of $0.05^{\prime \prime}$ from a $1^{\prime}$ split field of view, dynamic range required to the drive mechanism is around 2,400. This is much more relaxed than picking up the target from the full field of view of $\phi 6^{\prime}$ while also taking care of the orientation of the rays. The fact that the neighboring mechanisms never collide with each other also assures reliability without requiring complicated controls.

Physically dividing the fields also avoids requirement for large lens materials. The pick-off arms option requires some sort of conditioning of the rays, e.g., making a telecentric image plane, on the telescope field of view. At least it requires a dewar window of the physical size of the telescope field of view. On the 8-m class telescopes, only limited materials are available to cover the telescope field of view. On the other hand, the split fields option only requires optics of the size of the split fields. This becomes more important on the coming 30-m telescopes that will have physically larger telescope field of view. It also enables optimization of the optics according to position of position inside the telescope field of view.

\subsubsection{Microlens Array or Image Slicer}

Before delivering light to a spectrograph, the two-dimensional distribution of light has to be reconfigured so that each spectrum from each spatial element is dispersed and recorded by the FPA. We are considering two options, MLA and image slicer made of metal, to realize the reconstruction of the spatial elements to be fed to the spectrograph. The MLA option is more durable than the image slicer option however requires more number of FPA pixels to avoid cross talk between spectra from different spatial elements. Fabrication of both MLAs and image slicers can be expensive.

Microlens array On the image plane, an MLA is placed to image the entrance pupil of each spatial element onto the object plane of the spectrograph. There, discrete images of the entrance pupil is formed. The spectrograph disperses the light to a direction that allows the spectral trace from each spatial element passes through 


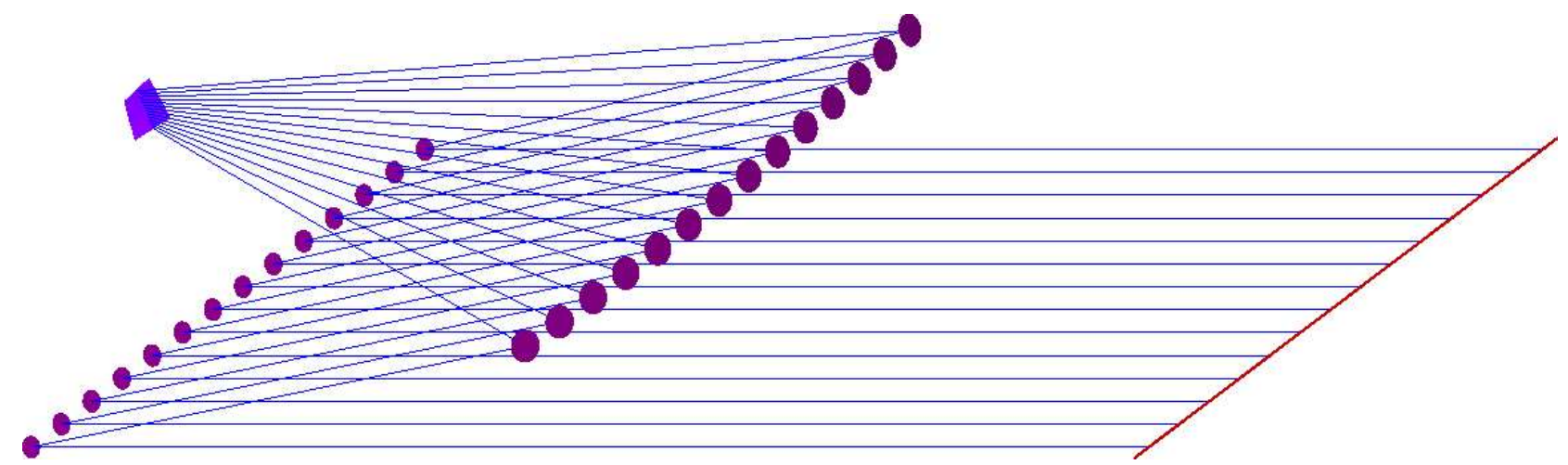

Figure 6. An example design of image slicer. Light from the telescope (not shown) comes from the right and imaged onto the slices of flat mirrors at the top left. A set of flat mirrors at the middle and a set of spherical mirrors at the bottom left re-images the field onto the pseudo slit at the right bottom while aligning orientation of the rays.

the gap between the other traces from the other spatial element. With an MLA inserted into the optical path, layout of the optics remains simple.

Filling factor of effective area inside a sub-field can be enough in spite of gaps between microlenses in an array. Magnification of the re-imaging optics can be adjusted to make each microlens big so that the gaps are negligible. Although it is possible to fabricate MLAs made of infrared transmissive materials like fused silica, it still costs some amount of money.

Separation of the spectral tracks on the FPA is important. It should be noted that adjacent tracks on the FPA are not from adjacent spatial elements. Moreover, light of different wavelengths from different spectral tracks arrives neighboring pixels of the FPA. Therefore, separation of spectral tracks on the FPA is important to avoid cross-talk between different spatial elements and different wavelengths.

With the simple layout of optics, MLA option is better than image slicer option when enough number of pixels on the FPA(s) can be afforded to keep separation of the spectral tracks.

Image slicer Image slicer is a set of mirrors laid out so that the two-dimensional image is sliced into strips and arranged onto an one-dimensional pseudo slit of a spectrograph. Figure 6 shows an example design of an image slicer.

Image slicers for infrared are practically reflective optics. As seen in Figure 6, layout and alignment of the optics can be complicated.

For each strip of the two-dimensional image on the pseudo slit, and thus on the spectrogram, light arriving adjacent pixels on the FPA is from adjacent spatial elements at the same wavelength. Therefore, gap between the spectral traces is only required between the sliced strips of the two-dimensional image. The pixels on an FPA is used more effectively with an image slicer than with an MLA as an IFU.

Image slicers for NIR would be able to be fabricated from a block of metal with numerically controlled grinding machines. This is expected to reduce costs than stacking polished glass plates to fabricate an image slicer.

\subsection{Adaptive Optics}

To take the full advantage of the multiple IFU, it is desirable that ultimately at least modest performance of MOAO is incorporated in CIRMOS. Adaptive optics is an indispensable part of most future infrared instrumentation for ground-based telescopes, yet there are still many technologies that challenge developments. The configuration of CIRMOS, as it is conceived at this time, would be ideal to test and implement one of those technologies: MOAO as applied to each of the IFU fields. For CIRMOS, our goal is MOAO for seeing-improved performance, hopefully modest in comparison to multi-conjugate AO for wide-field or extreme AO for highcontrast point spread function (PSF) for limited field of view. Exploration of tomographic approach we are 
Table 2. Phased development of MOAO. Columns show configurations and delivered image qualities for different phases.

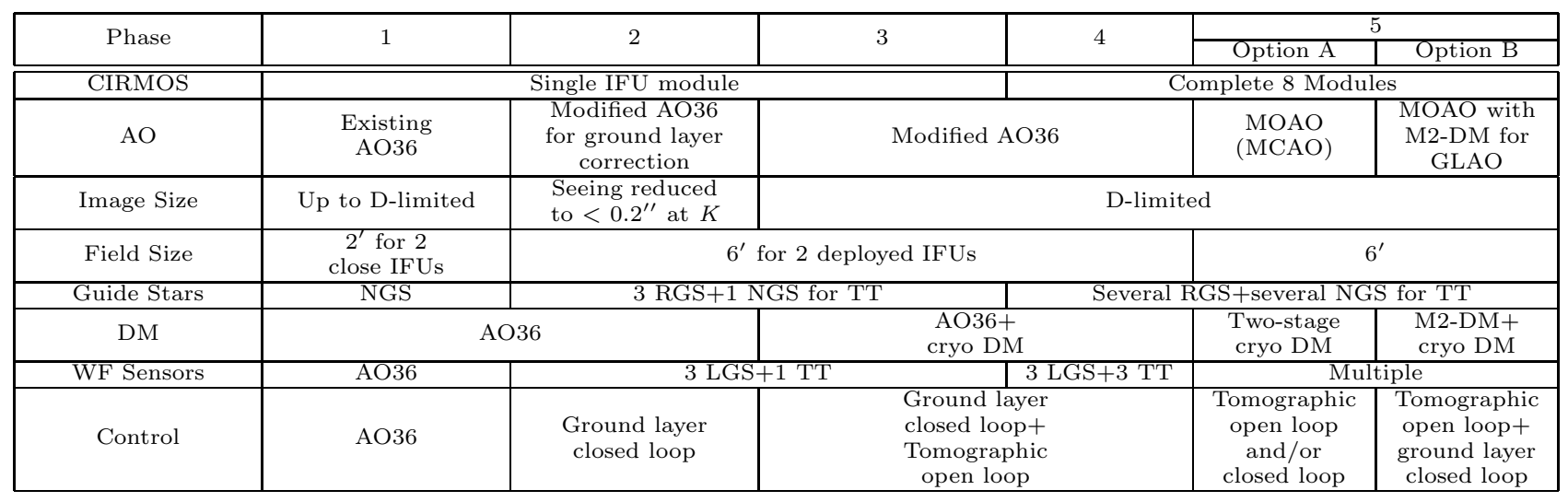

considering is the largest challenge, which includes, for example, algorithm development for open loop control. It may, however, relax hardware requirements such as strokes of DMs that need to be installed inside individual cryogenic modules with price to pay for PSF until the requirements are met in the future.

We plan to implement the MOAO capability in phases concurrently with the CIRMOS science modules, which are summarized in Table 2. The modularized instrument configuration concept in Figure 4 is to allow attaching several $\mathrm{AO}$ wave front sensor modules around the central focal plane module. The configuration may allow installation of LGS units, if they are compact enough, in which case the laser beams may travel through the telescope itself.

Phases $1-4$ are flexibly linked to the construction of a set of scientific modules for a single IFU. During these phases, we plan to use the first generation AO system, dubbed "AO36", at the Cassegrain focus of Subaru Telescope. The DM with 36 bimorph elements and its relay optics are mounted above the instrument mounting flange. It could be used with its original wave front sensor module in Phase 1 to feed an IFU. The system provide PSF reduced to diffraction limited performance over a $2^{\prime} \mathrm{FoV}^{7}$ in $J, H$ and $K$-bands. Phase 2 would concentrate on extending the system with LGSs to increase the sky coverage. Rayleigh LGS (RLGS) demonstrated to be a sufficient alternative for the much more expensive and complicated sodium LGS. In this phase we would still utilize the AO36 DM, now driven by 3 RLGS wavefront sensors for higher order correction and the AO36 sensor or a new NGS sensor for tip-tilt (TT) correction. Such combination will provide ground layer corrected seeing reduction within the $6^{\prime}$ FoV. Because of the limited number of TT stars, the PSF varies over the full FoV, but will be homogeneous enough within the much smaller IFU field. First experience with tomographic reconstruction could already be gained in this phase. Once we have cryo-DMs, possibly MEMS type, that can be installed in the beam delivery module of the IFU section (Phase 3), we can start with testing open loop controlled tomographic reconstruction in a woofer-tweeter style: the AO36 DM takes care of the low order correction with large stroke (woofer) over the full acquisition field in closed loop, while the cryo-DM corrects for higher order aberrations (tweeter) over the smaller IFU field in an open loop. If the stroke of the cryo DM is large enough one could already consider to move on to a purely tomographic approach in open loop. Phase 4 adds more TT wavefront sensors to provide homogeneous correction to all IFUs. Finally, in Phase 5 the AO36 DM would be removed and substituted either by an AO secondary mirror on the telescope (Option B) or in each IFU field a further large stroke low order cryo-DM (Option A), if available.

\subsection{Modular Construction}

For any instrument project, managing project schedule and cost is the key for its success. CIRMOS addresses them by modular construction and extensive prototyping. The multiple-IFU configuration will be implemented only after the entire concept is verified by extensive prototyping including testing on the telescope. The instrument packaging, however, is designed by anticipating this multiplication from the start. We achieve this by modular construction of the instrument as show in Figure 4. The package consists of four major modules for 
science modules: focal plane (FP) module, beam delivery (BD) modules, IFU modules, and an array detector (AD) module.

For prototyping, only the FP and AD modules and one each of the BD and IFU modules will be constructed. Only after the single BD and IFU module prototyping is verified successful on the telescope, multiplication to the final configuration should proceed. Additional AO units could be also arranged around the FP module as the AO systems are phased in as described in $\S 3.2$.

If final configuration has $8 \mathrm{BD}$ modules as an example mentioned earlier, the FP module will be a cylinder with 16 sides. Every other side will be connected through a gate valve to each of eight BD modules. The gate valve scheme was used in Subaru MOIRCS instrument separating its main cryostat and the mask carousel cryostat, the latter of which is frequently cycled between cryogenic operation temperature and room temperature for mask exchange by closing the gate valve without any problem. By connecting CIRMOS modules with gate valves it is not only convenient for the prototyping-then-multiplication scheme, but would serve to isolate modules for easy services later, too.

In the pick-off mirror option, the FP module could remain warm, while the BD module has to be cooled as does the IFU modules. By opening the gate valves the vacuum is shared between the central FP module and the $\mathrm{BD}$ modules. The pick-off mirror arm needs to be retracted entirely inside the BD module for closing the gate valves for module isolation. The sampled beam travels through the BD and IFU modules vertically and exits from the IFU module horizontally into the AD module, where the spatially formatted spectra are focused onto an array looking toward the IFU module. The AD module will be again 16-sided cylinder located directly below the FP module, and connected to each of IFU modules again with gate valves. Only one array will be installed during the prototyping phase. After the verification, the rest of seven arrays would be installed looking outwards to each of the IFU modules. It is our intention to connect the optical components in the BD and IFU module thermally with thermal switches that cross through the gate valves to cool with a single closed cycle cooler. The thermal switches closes only when the gate valves are open. The gradual closing of the switch allows controlled cooling of the component so that thermal shocks to optical components are minimized. Also each module on both sides of the gate valves has its own pumping port so that the vacuum of the other sides can be controlled before the gate valve is opened.

As AO technology becomes more sophisticated, it also becomes more challenging to construct a universal AO that can be shared by multiple instruments. It is our plan for CIRMOS to incorporate an AO system uniquely optimized to CIRMOS, and to package it as much as possible within the CIRMOS instrument frame. Thus, the concept of modularized construction of the AO system is also critical to the project. It would also allow independent teams to contribute to the project with minimum interference to each other.

\section{CONCLUSION}

We presented a conceptual outline of a Multi-Object 3D Spectrograph for the Subaru Telescope (CIRMOS). Our strategy to build such instrument is by "divide and conquer" to solve engineering challenges step by step and to push the budgetary burden to the end of the project. Therefore, we split the instrument in several different modules: focal plane module, AO module, beam delivery module, IFU module, and detector module. The different modules are connected with gate valves to allow the sequential assembly and provide easy access for maintenance later on. We discussed several choices to be made, i.e. focus, where we prefer the Cassegrain, pick off arms vs. split fields, microlens array vs. image slicers. Starting with one or two IFU modules we want to enhance the AO performance and sky coverage with time. In the beginning we plan to re-activate the existing Subaru AO36 system and upgrade it with lasers, new sensors and finally new deformable mirrors over different project phases. The final implementation goals on up to 8 IFUs with cryogenic deformable mirrors each and utilizing Rayleigh Laser Guide Stars for tomographic reconstruction.

\section{REFERENCES}

[1] Tomono, D., Weisz, H., and Hofmann, R., "Fiber IFU unit for the second generation VLT spectrograph KMOS," in [Instrument Design and Performance for Optical/Infrared Ground-based Telescopes. Edited by Iye, Masanori; Moorwood, Alan F. M. Proceedings of the SPIE, Volume 4841, pp. 390-397 (2003).], Iye, M. 
and Moorwood, A. F. M., eds., Presented at the Society of Photo-Optical Instrumentation Engineers (SPIE) Conference 4841, 390-397 (Mar. 2003).

[2] Blain, A. W., Kneib, J.-P., Ivison, R. J., and Smail, I., "Deep Counts of Submillimeter Galaxies," ApJ 512, L87-L90 (Feb. 1999).

[3] Daddi, E., Cimatti, A., and Renzini, A., "EROs and the formation epoch of field ellipticals," $A \& B A$ 362, L45-L48 (Oct. 2000).

[4] Bershady, M. A., Lowenthal, J. D., and Koo, D. C., "Near-Infrared Galaxy Counts to J and K $~ 24$ as a Function of Image Size," ApJ 505, 50-73 (Sept. 1998).

[5] Totani, T., Yoshii, Y., Maihara, T., Iwamuro, F., and Motohara, K., "Near-Infrared Faint Galaxies in the Subaru Deep Field: Comparing the Theory with Observations for Galaxy Counts, Colors, and Size Distributions to K 24.5," ApJ 559, 592-605 (Oct. 2001).

[6] Giacconi, R., Rosati, P., Tozzi, P., Nonino, M., Hasinger, G., Norman, C., Bergeron, J., Borgani, S., Gilli, R., Gilmozzi, R., and Zheng, W., "First Results from the X-Ray and Optical Survey of the Chandra Deep Field South," ApJ 551, 624-634 (Apr. 2001).

[7] Oya, S., Takato, N., Takami, H., Hayano, Y., Iye, M., Terada, H., Murakawa, K., Minowa, Y., Hattori, M., Watanabe, M., Kamata, Y., Kanzawa, T., Kane, T., and Gaessler, W., "Subaru adaptive optics system after two years of open use," in [Advancements in Adaptive Optics. Edited by Domenico B. Calia, Brent L. Ellerbroek, and Roberto Ragazzoni. Proceedings of the SPIE, Volume 5490, pp. 409-420 (2004).], Bonaccini Calia, D., Ellerbroek, B. L., and Ragazzoni, R., eds., Presented at the Society of Photo-Optical Instrumentation Engineers (SPIE) Conference 5490, 409-420 (Oct. 2004). 